\begin{tabular}{lc}
\hline SCIENCE \& TECHNOLOGY \\
Journal homepage: http://www.pertanika.upm.edu.my/ \\
\hline PERTANIKA
\end{tabular}

\title{
A New Method to Estimate Peak Signal to Noise Ratio for Least Significant Bit Modification Audio Steganography
}

\author{
Muhammad Harith Noor Azam ${ }^{1}$, Farida Ridzuan ${ }^{1,2 *}$ and M Norazizi Sham \\ Mohd Sayuti ${ }^{3}$ \\ ${ }^{1}$ Faculty of Science and Technology, Universiti Sains Islam Malaysia, Bandar Baru Nilai, Nilai 71800 USIM, \\ Negeri Sembilan, Malaysia \\ ${ }^{2}$ CyberSecurity and Systems Research Unit, Faculty of Science and Technology, Universiti Sains Islam Malaysia, \\ Nilai 71800 USIM, Negeri Sembilan, Malaysia \\ ${ }^{3}$ Intelligent Cyber-physical System Research Group(iCPS), Faculty of Engineering and Built Environment, \\ Universiti Sains Islam Malaysia, Nilai, 71800 USIM, Negeri Sembilan, Malaysia
}

\begin{abstract}
Audio steganography is implemented based on three main features: capacity, robustness, and imperceptibility, but simultaneously implementing them is still a challenge. Embedding data at the Least Significant Bit (LSB) of the audio sample is one of the most implemented audio steganography methods because the method will give high capacity and imperceptibility. However, LSB has the lowest robustness among all common methods in audio steganography. To cater to this problem, researchers increased the depth of the embedding level from fourth to sixth and eighth LSB level to improve its robustness feature. However, consequently, the imperceptibility feature, which is commonly measured by Peak Signal to Noise Ratio (PSNR), is reduced due to the trade-off between imperceptibility and robustness. Currently, the lack of study on the estimation of the PSNR for audio steganography has caused the early assessment of the imperceptibility-robustness tradeoff difficult. Therefore, a method to estimate PSNR, known as PSNR Estimator (PE), is introduced to enable early evaluation of imperceptibility feature for each stego-file

ARTICLE INFO

Article history:

Received: 15 June 2021

Accepted: 29 September 2021

Published: 10 January 2022

DOI: https://doi.org/10.47836/pjst.30.1.27

$\overline{\text { E-mail addresses: }}$

harithazam.official@gmail.com (Muhammad Harith Noor Azam)

farida@usim.edu.my (Farida Ridzuan)

azizi@usim.edu.my (M Norazizi Sham Mohd Sayuti)

* Corresponding author

produced by the audio steganography, which is important for the utilisation of embedding. The proposed PE estimates the PSNR based on the pattern collected from the embedment at different levels. From the evaluation, the proposed method has $99.9 \%$ of accuracy in estimating PSNR values at different levels. In comparison with the Mazdak Method,
\end{abstract}


the proposed method performs better in all situations. In conclusion, the proposed PE can be used as a reference for embedding and further reducing the calculation complexity in finding the feasible value to minimise the trade-off between robustness and imperceptibility.

Keywords: Audio steganography, Least Significant Bit, peak-signal-to-noise-ratio estimator, trade-off

\section{INTRODUCTION}

This Audio steganography is a method to conceal the secret message by using some method to modify the audio so that only the sender and the intended recipient knows (Alsabhany et al., 2018; Hameed, 2018; Hameed et al., 2019; Jayapandiyan et al., 2020; Sapra, 2016). It exploits Human Auditory System (HAS) to achieve secure communication (Yu et al., 2020). The common audio files used in audio steganography are wave file (.wav) and MPEG-1 Audio Layer III (.mp3) (Sun et al., 2012; Wakiyama et al., 2010).

For any audio steganography method to be successfully implemented, it is important to consider three features: capacity, imperceptibility and robustness (Alsabhany et al., 2020; Amirtharajan \& Rayappan, 2013; Hameed, 2018; Zumchak, 2016). First, capacity refers to the amount of secret data embedded inside a cover file (Somani \& Madhu, 2015; Srivastava \& Rafiq, 2012). Next, the imperceptibility means that the furtive data should not be detected in humans, although there is a difference between cover file and stego-file audio (Somani \& Madhu, 2015; Srivastava \& Rafiq, 2012). Lastly, robustness refers to the ability of the embedded data to withstand attacks (Somani \& Madhu, 2015; Zumchak, 2016). Although audio steganography needs all these features in top conditions simultaneously, there are always trade-offs between all these three features, such as between capacity and robustness (Bhowal et al., 2017; Kumar, 2016), capacity and imperceptibility(Ali et al., 2017; Alsabhany et al., 2019) and lastly, imperceptibility and robustness (Gopalan \& Fu, 2015; Xue et al., 2018). The trade-off relationship between audio steganography features is shown in Figure 1. Total disregard for any feature may lead to an unbalanced and impractical audio steganography method (Ali et al., 2017; Gopalan, 2018).

The most common existing audio steganography methods are phase coding, Least Significant Bit (LSB) modification, echo hiding, parity coding, wavelet domain and spread spectrum (Mazdak et al., 2012). This research focuses on the LSB modification because

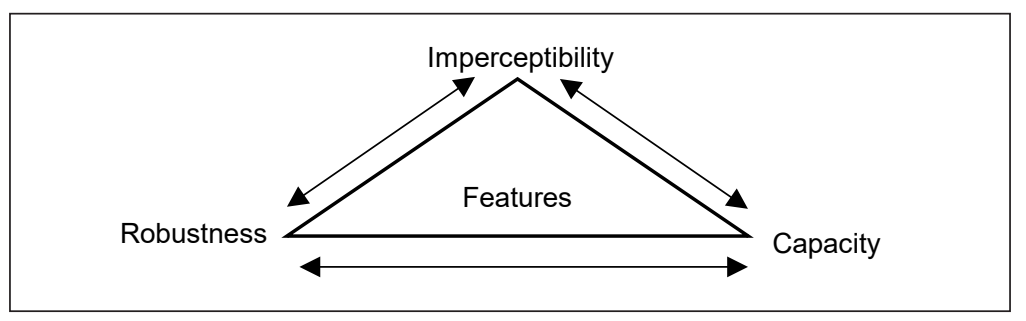

Figure 1. Trade-off relationship among audio steganography features 
of its simplicity, flexibility to modification, efficiency, high imperceptibility and capacity (Asad et al., 2011; Djebbar et al., 2011; Tayel et al., 2016; Mazdak et al., 2012). However, although it has numerous advantages, it has low robustness features and is easy to extract (Cvejic \& Seppänen, 2005; Kanhe et al., 2015; Meligy et al., 2016). Therefore, researchers proposed to embed the data at a higher level of LSB to increase the robustness features of audio steganography (Ahmed et al., 2010; Priyanka et al., 2012; Cvejic \& Seppänen, 2004). However, by doing so, the imperceptibility feature in the LSB method is reduced.

The imperceptibility feature of an audio steganography method is commonly calculated using PSNR. However, to measure the PSNR, a stego-file needs to be produced from the embedding process. In order to find the feasible results that can be used to minimise the trade-off between imperceptibility and robustness feature, the process of embedding the secret data at a different level of LSB and comparing the PSNR value for each stego-file produced is needed. These processes are time-consuming. Therefore, PE is introduced.

The method to estimate the PSNR, also known as PE, will enable early evaluation on imperceptibility feature for each stego-file produced by the audio steganography that can be used as a reference for utilisation of embedding. Additionally, in a real-time application, computational complexity is crucial to ensure an audio steganography implementation (Mazdak et al., 2012). Therefore, PE can avoid repetitive embedding processes in finding the feasible solution for the trade-off, further reducing the computational complexity. Furthermore, we can avoid bad stego-file from being produced, which improves audio steganography efficiency.

The structure of the paper is as follows: the related works are presented in section 2; the proposed method is presented in section 3 ; the expected results are presented in section 4 ; and finally, the conclusion and future work are presented in section 5.

\section{RELATED WORK}

Most of the existing research on audio steganography targets only one or two features and discards the other. Hence, PSNR calculation is only carried out at the final stage. Therefore, although several advantages can be achieved by estimating the PSNR, only one research has proposed on PSNR estimation in audio steganography. Mazdak et al. (2012) proposed a PSNR estimation method based on linear interpolation formula. The method uses PSNR value and bit per sample (BPS) rate on existing stego-file to estimate PSNR value of new stego-file that embed different bps rate. This method tries to tackle the trade-off between imperceptibility and capacity features of audio steganography.

$\mathrm{PE}$ is also used in image steganography for similar purposes. For example, Li et al. (2014) had proposed PSNR estimation based on Mean Squared Error (MSE) value. It is used to compare the level of distortion between the original signal and the stegofile (Chadha et al., 2013). Suppose $x=\left\{x_{i} \mid i=1,2,3, \ldots, N\right\}$ is the original signal and 
$y=\left\{y_{i} \mid i=1,2,3, \ldots, N\right\}$ is the stego-file signal and where $\mathrm{N}$ is the number of signal samples and $x_{i}$ and $y_{i}$ are the value of $\mathrm{i}^{\text {th }}$ samples in $x$ and $y$, respectively. The MSE formula is calculated using Equation 1:

$$
\operatorname{MSE}(x, y)=\frac{1}{N} \sum_{1}^{N}\left(x_{i}-y_{i}\right)^{2}
$$

This estimation is also calculated based on bit per sample. However, it is mentioned that the method works best when the BPS rate is less than four because of the size of bit depth in the image sample that is considered small when compared to the audio. This estimation method reduces the expansion of the stego-file needed for the shadow image. The main focus of this method is to improve the imperceptibility feature of image steganography instead of tackling the existing trade-off.

Based on the previous research, it is shown that there is a lack of research conducted for estimating the PSNR value in audio steganography. In addition, the lack of a PSNR estimator focuses on determining the PSNR value to solve the trade-off. The only PSNR estimator in audio steganography is focused on determining the efficiency of a steganography technique for the trade-off between capacity and imperceptibility. Therefore, this research tries to provide a new method for estimating the PSNR value at the different levels of LSB of audio steganography based on PSNR patterns defined from embedding at a different level of LSB to cater to different types of trade-off, which is between imperceptibility and robustness.

\section{METHODOLOGY}

This paper proposes a method to estimate the PSNR value for audio stego-file based on the patterns observed from the PSNR values obtained from embedding at a different level of LSB. A series of experiments are conducted to find patterns in PSNR values. The first experiment was conducted on finding PSNR value at the lowest level of LSB for different sizes of embedded data and the number of audio samples. The second experiment was conducted on finding PSNR values at a different level of LSB for different sizes of embedded data and the number of audio samples. Finally, the new method for estimating PSNR value is proposed based on the patterns from the PSNR values obtained at a different level of LSB embedding.

\section{PSNR of One Bit per Sample at the First LSB}

The objective of this experiment is to observe the similarity of the PSNR value at the lowest level of LSB. The PSNR values between all the stego-file produced should be almost identical to ensure the validity of the pattern. Ten different cover audios ranging 
from one to ten seconds with 16-bit depth and $44.1 \mathrm{kHz}$ mono are selected from various speech and music .wav audio files to ensure the diversity of the experiment's samples. The ratio of the size of data embedded to number of audio sample is approximately one to two. For each cover audio, a customised size of data is embedded at the first level of LSB of cover audio samples, and the PSNR values for each stego-file produced are recorded. Cover audio name, a total of cover audio samples, size of data embedded and obtained PSNR are tabulated in Table 1.

Table 1 shows that the PSNR for all tested cover audio is between a range of 96.32 $\mathrm{dB}$ and $96.34 \mathrm{~dB}$, which indicates that PSNR values are almost the same at the lowest level of LSB.

Table 1

Obtained PSNR for one bit per sample at first level LSB

\begin{tabular}{cccc}
\hline Cover Audio & Cover Audio Samples & Size of Data (bit) & PSNR \\
\hline Music 1.wav & 44100 & 22050 & 96.328 \\
Music 2.wav & 132300 & 66150 & 96.317 \\
Music 3.wav & 220500 & 110250 & 96.331 \\
Music 4.wav & 352800 & 176400 & 96.328 \\
Music 5.wav & 441000 & 220500 & 96.336 \\
Speech 1.wav & 44100 & 22050 & 96.292 \\
Speech 2.wav & 132300 & 66150 & 96.343 \\
Speech 3.wav & 220500 & 110250 & 96.318 \\
Speech 4.wav & 352800 & 176400 & 96.323 \\
Speech 5.wav & 441000 & 220500 & 96.324 \\
\hline
\end{tabular}

\section{PSNR of One Bit per Sample at Different Level LSB}

The objective of this experiment is to find the pattern among the PSNR value produced from embedding at a different level of LSB. Therefore, the data is embedded at a different level of LSB. The second, fourth, sixth, seventh and eighth levels of LSB are selected. This experiment is implemented by embedding the data at a different level of LSB of cover audio sample accordingly. Then, the PSNR value for each stego-file is recorded. Cover audio names and obtained PSNR at different LSB levels are tabulated in Table 2.

Based on Table 2, the stego-file produced shows the identical value at all embedding levels. In addition, significant and identical decrement for PSNR value from the lowest level of LSB to the highest level of LSB is shown in all audios. Therefore, it can be concluded that there is a pattern in the difference of PSNR value that can be used to estimate the PSNR at a different level. 
Table 2

Obtained PSNR of one bit per sample at different level LSB

\begin{tabular}{lcccccc}
\hline \multirow{2}{*}{ Cover Audio } & \multicolumn{7}{c}{ PSNR Obtained } \\
\cline { 2 - 7 } & $1^{\text {st }}$ level & $2^{\text {nd }}$ level & $4^{\text {th }}$ level & $6^{\text {th }}$ level & $7^{\text {th }}$ level & $8^{\text {th }}$ level \\
\hline Music 1.wav & 96.328 & 90.330 & 78.256 & 66.215 & 60.203 & 54.186 \\
Music 2.wav & 96.317 & 90.289 & 78.253 & 66.221 & 60.216 & 54.183 \\
Music 3.wav & 96.331 & 90.306 & 78.243 & 66.226 & 60.192 & 54.171 \\
Music 4.wav & 96.328 & 90.293 & 78.263 & 66.253 & 60.208 & 54.195 \\
Music 5.wav & 96.336 & 90.299 & 78.274 & 66.218 & 60.226 & 54.177 \\
Speech 1.wav & 96.292 & 90.315 & 78.260 & 66.214 & 60.150 & 54.193 \\
Speech 2.wav & 96.343 & 90.322 & 78.264 & 66.210 & 60.191 & 54.210 \\
Speech 3.wav & 96.318 & 90.289 & 78.277 & 66.233 & 60.231 & 54.182 \\
Speech 4.wav & 96.323 & 90.288 & 78.271 & 66.229 & 60.205 & 54.190 \\
Speech 5.wav & 96.324 & 90.308 & 78.269 & 66.246 & 60.205 & 54.213 \\
\hline
\end{tabular}

\section{Method on Estimating PSNR Value based on Pattern}

In order to estimate the PSNR value for a different level of LSB, the differences between the PSNR value from Table 2 are used as a basis. The PSNR difference at different levels of LSB is shown in Table 3.

Table 3

Obtained PSNR difference at different level of LSB

\begin{tabular}{lccccc}
\hline \multirow{2}{*}{ Cover Audio } & \multicolumn{5}{c}{ PSNR Difference at Different Level } \\
\cline { 2 - 5 } & $1^{\text {st }} \& 2^{\text {nd }}$ level & $2^{\text {nd }} \& 4^{\text {th }}$ level & $4^{\text {th }} \& 6^{\text {th }}$ level & $6^{\text {th }} \& 7^{\text {th }}$ level & $7^{\text {th }} \& 8^{\text {th }}$ level \\
\hline Music 1.wav & 5.998 & 12.074 & 12.041 & 6.012 & 6.017 \\
Music 2.wav & 6.028 & 12.036 & 12.032 & 6.005 & 6.033 \\
Music 3.wav & 6.025 & 12.063 & 12.017 & 6.034 & 6.021 \\
Music 4.wav & 6.035 & 12.03 & 12.01 & 6.045 & 6.013 \\
Music 5.wav & 6.037 & 12.025 & 12.056 & 5.992 & 6.049 \\
Speech 1.wav & 5.977 & 12.055 & 12.046 & 6.064 & 5.957 \\
Speech 2.wav & 6.021 & 12.058 & 12.054 & 6.019 & 5.981 \\
Speech 3.wav & 6.029 & 12.012 & 12.044 & 6.002 & 6.049 \\
Speech 4.wav & 6.035 & 12.017 & 12.042 & 6.024 & 6.015 \\
Speech 5.wav & 6.016 & 12.039 & 12.023 & 6.041 & 5.992 \\
\hline
\end{tabular}

Table 3 shows the obtained PSNR values for ten audio files at different embedding levels. Since the differences are almost identical, ranging from 5.977 to 6.049 at one different level and ranging from 12.01 to 12.074 at two different levels, the average can create a constant in developing a formula for PSNR estimation.

In order to collect the average of PSNR differences between each level, the value of the difference at two levels of embedding should be divided by two to get the average difference 
in PSNR value at one difference level. Therefore, a new table is tabulated to display all the differences. Table 4 shows the difference in the PSNR values at all different levels of LSB.

Table 4

Obtained PSNR difference at all level of LSB

\begin{tabular}{lccccccc}
\hline & \multicolumn{7}{c}{ PSNR Difference at Different Level } \\
\cline { 2 - 7 } Cover Audio & $\begin{array}{c}1^{\text {st }} \& 2^{\text {nd }} \\
\text { level }\end{array}$ & $\begin{array}{c}2^{\text {nd }} \& 3^{\text {rd }} \\
\text { level }\end{array}$ & $\begin{array}{c}3^{\text {rd }} \& 4^{\text {th }} \\
\text { level }\end{array}$ & $\begin{array}{c}4^{\text {th }} \& 5^{\text {th }} \\
\text { level }\end{array}$ & $\begin{array}{c}5^{\text {th }} \& 6^{\text {th }} \\
\text { level }\end{array}$ & $\begin{array}{c}6^{\text {th }} \& 7^{\text {th }} \\
\text { level }\end{array}$ & $\begin{array}{c}7^{\text {th }} \& 8^{\text {th }} \\
\text { level }\end{array}$ \\
\hline Music 1.wav & 5.998 & 6.037 & 6.037 & 6.021 & 6.021 & 6.012 & 6.017 \\
Music 2.wav & 6.028 & 6.018 & 6.018 & 6.016 & 6.016 & 6.005 & 6.033 \\
Music 3.wav & 6.025 & 6.032 & 6.032 & 6.009 & 6.009 & 6.034 & 6.021 \\
Music 4.wav & 6.035 & 6.015 & 6.015 & 6.005 & 6.005 & 6.045 & 6.013 \\
Music 5.wav & 6.037 & 6.013 & 6.013 & 6.028 & 6.028 & 5.992 & 6.049 \\
Speech 1.wav & 5.977 & 6.028 & 6.028 & 6.023 & 6.023 & 6.064 & 5.957 \\
Speech 2.wav & 6.021 & 6.029 & 6.029 & 6.027 & 6.027 & 6.019 & 5.981 \\
Speech 3.wav & 6.029 & 6.006 & 6.006 & 6.022 & 6.022 & 6.002 & 6.049 \\
Speech 4.wav & 6.035 & 6.009 & 6.009 & 6.021 & 6.021 & 6.024 & 6.015 \\
Speech 5.wav & 6.016 & 6.02 & 6.02 & 6.012 & 6.012 & 6.041 & 5.992 \\
\hline
\end{tabular}

After calculating the difference value at each level, the average difference for PSNR value between each level is 6.02. Hence, a novel formula is proposed, which is based on the average differences retrieved from the pattern shown. The formula is as in Equation 2:

$$
y=x-\left(6.02 *\left(l_{n}-l_{x}\right)\right)
$$

where $y$ is the expected PSNR at a different level, while $x$ is the initial PSNR value at any level, while 6.02 is a constant calculated from the average differences between one upper level and one lower level. Finally, $l_{n}$ is the new embedding LSB level, while $l_{x}$ is the initial LSB level. The new formula can estimate the PSNR value for stego-file at a different level of LSB embedding. Figure 2 shows the flowchart of the proposed estimation method.

The estimation process starts by selecting the cover audio and secret message that needs to be hidden. Next, the secret message is embedded into one of the least

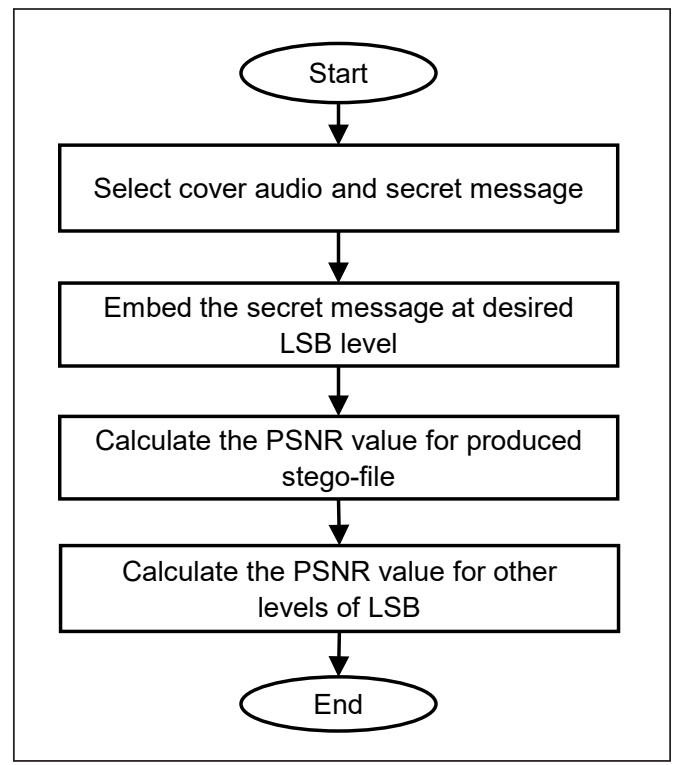

Figure 2. Proposed estimation method flowchart 
significant bit levels of the cover audio sample, and modified cover audio is compiled into the stego-file. The stego-file is then evaluated by calculating its PSNR value. Finally, the PSNR value for other levels of LSB can be measured using Equation 2.

\section{EVALUATION AND DISCUSSION}

An accuracy test is carried out to evaluate the proposed method. The average accuracy for the method is calculated using the Equation 3:

$$
a=100 \%-\left(\frac{d}{v}\right) * 100 \%
$$

where a is the average accuracy, $d$ is the average absolute difference between estimated PSNR and actual PSNR, and $\mathrm{v}$ is the average of actual PSNR value.

All covers for this evaluation are collected from www.freesound.org. They have 44.1 KHz for sample rate, mono channel, wav file format and has 16-bit depth. In addition, speech, music, and silence type audio ranged, which has 1, 3, 5, 8, and 10 seconds are used to give a better view on the capabilities of the method. The number of secret message bits embedded in the cover for this test is at around $50 \%$ from the audio sample.

This test compares the estimation of PSNR value for fifteen (15) stego-file at different embedding levels against the actual PSNR value of this stego-file. Furthermore, it estimates two different levels of embedding, which are at the third level of LSB, and the seventh level of LSB, for a better view of the method's performance. Therefore, the proposed Equation 2 is used, and the result for the estimation stego-file PSNR from third level embedding is recorded in Table 5, while the result for the estimation stego-file PSNR from seventh level embedding is recorded in Table 6 .

Based on Table 5, the average difference between the estimation and the actual result is 0.0184 . For the individual difference, the differences vary between one to the others because of the difference of cover audio files and secret data used. Using different cover audio files and secret data will generate different numbers of bits flipping, leading to the error during the embedding process. Furthermore, if the size of the secret message is big, more modifications will be made to the cover audio sample bit; hence higher error is introduced. In this round, this method can estimate the PSNR value with $99.978 \%$ accuracy.

Based on Table 6, the average difference between the estimation and the actual result is 0.0118 . The difference is quite similar to the result from Table 5, and both values are considered small. In this round, this method can estimate the PSNR value with 99.98\% accuracy.

Based on the result from Tables 5 and 6, this method can estimate the PSNR value with high accuracy. Furthermore, the result shows that the difference between estimation and the actual value is less than 0.1 at different levels. 
Table 5

Comparison between estimation and actual result of stego-file from $3^{\text {rd }}$ level embedding

\begin{tabular}{ccccc}
\hline Audio & $\begin{array}{c}\text { Obtained PSNR } \\
\text { for 4bps }\end{array}$ & $\begin{array}{c}\text { Estimated PSNR } \\
\text { for 3bps }\end{array}$ & $\begin{array}{c}\text { Actual PSNR for } \\
\text { 3bps }\end{array}$ & $\begin{array}{c}\text { Absolute } \\
\text { difference }\end{array}$ \\
\hline Music 1.wav & 78.3062 & 84.3262 & 84.3459 & 0.020 \\
Music 2.wav & 78.2817 & 84.3017 & 84.2983 & 0.003 \\
Music 3.wav & 78.2326 & 84.2526 & 84.2813 & 0.029 \\
Music 4.wav & 78.277 & 84.297 & 84.2893 & 0.008 \\
Music 5.wav & 78.2686 & 84.2886 & 84.3032 & 0.015 \\
Speech 1.wav & 78.2513 & 84.2713 & 84.2699 & 0.001 \\
Speech 2.wav & 78.2926 & 84.3126 & 84.2652 & 0.047 \\
Speech 3.wav & 78.3005 & 84.3205 & 84.2828 & 0.038 \\
Speech 4.wav & 78.2882 & 84.3082 & 84.2986 & 0.010 \\
Speech 5.wav & 78.2872 & 84.3072 & 84.2963 & 0.011 \\
Silence 1.wav & 78.2864 & 84.3064 & 84.3034 & 0.003 \\
Silence 2.wav & 78.3011 & 84.3211 & 84.3081 & 0.013 \\
Silence 3.wav & 78.3102 & 84.3302 & 84.3067 & 0.023 \\
Silence 4.wav & 78.3133 & 84.3333 & 84.3009 & 0.032 \\
Silence 5.wav & 78.3012 & 84.3212 & 84.2983 & 0.023 \\
\hline & & Average & 84.29654667 & 0.0184 \\
\hline
\end{tabular}

Table 6

Comparison between estimation and actual result of stego-file from $7^{\text {th }}$ level embedding

\begin{tabular}{ccccc}
\hline Audio & $\begin{array}{c}\text { Obtained PSNR at } \\
\text { 5th Level }\end{array}$ & $\begin{array}{c}\text { Estimated PSNR } \\
\text { at 7th Level }\end{array}$ & $\begin{array}{c}\text { Actual PSNR at } \\
\text { 7th Level }\end{array}$ & $\begin{array}{c}\text { Absolute } \\
\text { difference }\end{array}$ \\
\hline Music 1.wav & 72.2872 & 60.2472 & 60.1891 & 0.0581 \\
Music 2.wav & 72.2685 & 60.2285 & 60.2065 & 0.0220 \\
Music 3.wav & 72.2662 & 60.2262 & 60.2098 & 0.0164 \\
Music 4.wav & 72.2396 & 60.1996 & 60.2001 & 0.0005 \\
Music 5.wav & 72.2522 & 60.2122 & 60.2034 & 0.0088 \\
Speech 1.wav & 72.2784 & 60.2384 & 60.2687 & 0.0303 \\
Speech 2.wav & 72.2568 & 60.2168 & 60.2297 & 0.0129 \\
Speech 3.wav & 72.254 & 60.214 & 60.2186 & 0.0046 \\
Speech 4.wav & 72.2477 & 60.2077 & 60.211 & 0.0033 \\
Speech 5.wav & 72.2573 & 60.2173 & 60.2317 & 0.0144 \\
Silence 1.wav & 72.2784 & 60.2384 & 60.2372 & 0.0012 \\
Silence 2.wav & 72.3043 & 60.2643 & 60.2631 & 0.0012 \\
Silence 3.wav & 72.2952 & 60.2552 & 60.254 & 0.0012 \\
Silence 4.wav & 72.2981 & 60.2581 & 60.2569 & 0.0012 \\
Silence 5.wav & 72.2829 & 60.2429 & 60.2417 & 0.0012 \\
\hline & & Average & 60.2281 & 0.0118 \\
\hline
\end{tabular}


A comparison was made between the proposed method and Mazdak Method (Mazdak et al., 2012). Mazdak Method focuses on estimating PSNR at different bps rates to cater for the trade-off between capacity and imperceptibility. On the other hand, the proposed method focuses on estimating the PSNR value for one bit per sample at a different level to cater for the trade-off between robustness and imperceptibility. Both methods have different objectives, but the accuracy percentage between them can still be compared.

In order to make a comparison, the same audio used in the previous test is used. PSNR values for $3 \mathrm{bps}$ and $7 \mathrm{bps}$ embedding are estimated using Mazdak Method and compared against the actual PSNR value. The formulation used for Mazdak Method to give a brief idea is presented in Equation 4:

$$
y=y_{a}+\left(x-x_{a}\right) \frac{y_{b}-y_{a}}{x_{b}-x_{a}}
$$

where $x$ is a given bps rate, and $y$ is its PSNR. Hence, $x_{a}$ represents the bps rate for $a, x_{b}$ represents the bps rate for $b, y_{a}$ represents the PSNR of embedding with $a$ bps rate, and $y_{b}$ represents the PSNR of embedding with $b$ bps rate.

PSNR values from embedding with 4 bps and 2 bps of 15 audio files are recorded in Table 7. Equation 4 was used to estimate the PSNR value for $3 \mathrm{bps}$. The estimated PSNR value for $3 b p s$, actual PSNR value for 3 bps and absolute difference is recorded in Table 7.

PSNR values from embedding with $5 \mathrm{bps}$ and $8 \mathrm{bps}$ of 15 audio files are recorded in Table 8. The same Equation 4 was used to calculate PSNR to estimate the PSNR value for $7 \mathrm{bps}$. The estimated PSNR value for 7bps, actual PSNR value for 7bps and the absolute difference is recorded in Table 8 .

Based on Table 7, the average absolute difference between the estimation and the actual result is 0.2953 . Therefore, based on Equation 3, Mazdak Method can estimate the PSNR value with $99.646 \%$.

Based on Table 8 , the average absolute difference between the estimation and the actual result is 0.8844 , and the estimation accuracy is $98.474 \%$. The differences are higher than the estimation of PSNR value from embedding $3 \mathrm{bps}$ secret message, which indicates that the Mazdak method has lower accuracy when estimating PSNR for the embedding at higher bps.

Although Mazdak Method can estimate the PSNR value when embedding with different bps rates, the accuracy is reduced when estimating a higher bps rate. The proposed method obtained higher accuracy than Mazdak Method because the patterns for whole cases were collected and studied, producing stable estimation, which can be maintained around 99.9. 
Table 7

Comparison between estimation and actual result based on Mazdak Method (3bps)

\begin{tabular}{cccccc}
\hline Audio & $\begin{array}{c}\text { Obtained } \\
\text { PSNR for 4bps }\end{array}$ & $\begin{array}{c}\text { Obtained PSNR } \\
\text { for 2bps }\end{array}$ & $\begin{array}{c}\text { Estimated } \\
\text { PSNR for 3bps }\end{array}$ & $\begin{array}{c}\text { Actual PSNR } \\
\text { for 3bps }\end{array}$ & $\begin{array}{c}\text { Absolute } \\
\text { difference }\end{array}$ \\
\hline Music 1.wav & 77.6152 & 89.6416 & 83.6284 & 83.1304 & 0.498 \\
Music 2.wav & 77.7262 & 89.6785 & 83.70235 & 83.3395 & 0.363 \\
Music 3.wav & 77.7314 & 89.7031 & 83.71725 & 83.3806 & 0.337 \\
Music 4.wav & 77.7226 & 89.7036 & 83.7131 & 83.3746 & 0.338 \\
Music 5.wav & 77.7247 & 89.7238 & 83.72425 & 83.3948 & 0.329 \\
Speech 1.wav & 77.8145 & 89.7295 & 83.772 & 83.4211 & 0.351 \\
Speech 2.wav & 77.8175 & 89.7161 & 83.7668 & 83.3902 & 0.377 \\
Speech 3.wav & 77.8026 & 89.7277 & 83.76515 & 83.3936 & 0.372 \\
Speech 4.wav & 77.8228 & 89.7263 & 83.77455 & 83.4121 & 0.362 \\
Speech 5.wav & 77.8317 & 89.7342 & 83.78295 & 83.2441 & 0.539 \\
Silence 1.wav & 76.5157 & 89.7555 & 83.1356 & 83.2157 & 0.080 \\
Silence 2.wav & 76.5209 & 89.6912 & 83.10605 & 83.255 & 0.149 \\
Silence 3.wav & 76.5293 & 89.6974 & 83.11335 & 83.242 & 0.129 \\
Silence 4.wav & 76.5236 & 89.7243 & 83.12395 & 83.229 & 0.105 \\
Silence 5.wav & 76.5381 & 89.7478 & 83.14295 & 83.244 & 0.101 \\
\hline
\end{tabular}

Table 8

Comparison between estimation and actual result based on Mazdak Method (7bps)

\begin{tabular}{cccccc}
\hline Audio & $\begin{array}{c}\text { Obtained } \\
\text { PSNR for 5bps }\end{array}$ & $\begin{array}{c}\text { Obtained } \\
\text { PSNR for 8bps }\end{array}$ & $\begin{array}{c}\text { Estimated } \\
\text { PSNR for 7bps }\end{array}$ & $\begin{array}{c}\text { Actual PSNR } \\
\text { for 7bps }\end{array}$ & $\begin{array}{c}\text { Absolute } \\
\text { difference }\end{array}$ \\
\hline Music 1.wav & 71.0445 & 54.6277 & 60.09996667 & 59.0499 & 1.050067 \\
Music 2.wav & 71.2037 & 54.6757 & 60.18503333 & 59.1703 & 1.014733 \\
Music 3.wav & 71.2392 & 54.7271 & 60.23113333 & 59.2143 & 1.016833 \\
Music 4.wav & 71.2929 & 54.7314 & 60.2519 & 59.2527 & 0.9992 \\
Music 5.wav & 71.2967 & 54.7486 & 60.26463333 & 59.2424 & 1.022233 \\
Speech 1.wav & 71.2006 & 53.1268 & 59.1514 & 58.1282 & 1.0232 \\
Speech 2.wav & 71.1503 & 52.7786 & 58.9025 & 57.9722 & 0.9303 \\
Speech 3.wav & 71.2226 & 53.2252 & 59.22433333 & 58.4405 & 0.783833 \\
Speech 4.wav & 71.1999 & 53.2412 & 59.22743333 & 58.3946 & 0.832833 \\
Speech 5.wav & 71.2124 & 53.1347 & 59.1606 & 58.3311 & 0.8295 \\
Silence 1.wav & 69.1936 & 51.1402 & 57.158 & 56.3932 & 0.7648 \\
Silence 2.wav & 69.1907 & 51.1533 & 57.16576667 & 56.4313 & 0.734467 \\
Silence 3.wav & 69.1883 & 51.1632 & 57.17156667 & 56.4236 & 0.747967 \\
Silence 4.wav & 69.1746 & 51.1607 & 57.16533333 & 56.4134 & 0.751933 \\
Silence 5.wav & 69.1861 & 51.1646 & 57.17176667 & 56.4071 & 0.764667 \\
\hline
\end{tabular}




\section{CONCLUSION}

This paper proposes a new method to estimate the PSNR value of any LSB embedding level to cater to the trade-off between imperceptibility and robustness. PSNR values' pattern from embedding at a different level of LSB, which affect the robustness features, can be used to estimate the PSNR specifically for the imperceptibility feature of audio steganography. The estimated PSNR value generated based on the new method has an average of $99.9 \%$ accuracy compared to the actual embedding result at a different level of LSB throughout different embedding levels. The proposed method obtained higher accuracy when compared to Mazdak Method and only needed one actual PSNR value compared to Mazdak Method, which needs two actual PSNR values to operate. However, the focus of the proposed method is only on LSB modification. Hence, it is not suitable and accurate to implement the frequency domain in audio steganography.

To conclude, this method can be used as a utilisation tool to refrain from the repetition of embedding processes in finding the feasible solution to cater for the trade-off problem between imperceptibility and robustness features and further reducing the computational complexity of unnecessary repetitive embedding processes. Furthermore, the efficiency of an audio steganography method can be improved as any bad cover audio can be avoided from being produced. Future work that can be considered includes creating a steganography method based on this new estimation method to obtain a method with balanced features in terms of imperceptibility and robustness.

\section{ACKNOWLEDGEMENT}

This work is supported in part by the Ministry of Higher Education (MOHE) Malaysia under a research grant [USIM/FRGS/FST/055002/50117]. The authors would like to express their gratitude to Universiti Sains Islam Malaysia (USIM) and MOHE for the support and facilities provided.

\section{REFERENCES}

Ahmed, M. A., Kiah, L. M., Zaidan, B. B., \& Zaidan, A. A. (2010). A novel embedding method to increase capacity and robustness of low-bit encoding audio steganography technique using noise gate software logic algorithm. Journal of Applied Sciences, 10(1), 59-64. https://doi.org/10.3923/jas.2010.59.64

Ali, A. H., Mokhtar, M. R., \& George, L. E. (2017). Enhancing the hiding capacity of audio steganography based on block mapping. Journal of Theoretical and Applied Information Technology, 95(7), 1441-1448.

Alsabhany, A. A., Ridzuan, F., \& Azni, A. H. (2019). The adaptive multi-level phase coding method in audio steganography for confidential communication. IEEE Access, 7, 129291-129306. https://doi.org/10.1109/ ACCESS.2019.2940640 
Alsabhany, A. A., Ridzuan, F., \& Azni, A. H. (2018). An adaptive multi amplitude thresholds embedding algorithm for audio steganography. Malaysian Journal of Science Health \& Technology, 2 (Special Issue), 7-10. https://doi.org/10.33102/mjosht.v2i.43

Alsabhany, A. A., Ridzuan, F., Ridzuan, F., Azni, A. H., \& Azni, A. H. (2020). The progressive multilevel embedding method for audio steganography. In Journal of Physics: Conference Series (Vol. 1551, No. 1, p. 012011). IOP Publishing. https://doi.org/10.1088/1742-6596/1551/1/012011

Amirtharajan, R., \& Rayappan, J. B. B. (2013). Steganography - Time to time: A review. Research Journal of Information Technology, 5(2), 53-66. https://doi.org/10.3923/rjit.2013.53.66

Asad, M., Gilani, J., \& Khalid, A. (2011). An enhanced least significant bit modification technique for audio steganography. In International Conference on Computer Networks and Information Technology (pp. 143-147). IEEE Publishing. https://doi.org/10.1109/ICCNIT.2011.6020921

Bhowal, K., Sarkar, D., Biswas, S., \& Sarkar, P. P. (2017). A steganographic approach to hide secret data in digital audio based on XOR operands triplet property with high embedding rate and good quality audio. Turkish Journal of Electrical Engineering \& Computer Sciences, 25(3), 2136-2148. https://doi. org/10.3906/elk-162-267

Chadha, A., Satam, N., \& Sood, R. (2013). An efficient method for image and audio steganography using least significant bit (LSB) substitution. International Journal of Computer Applications, 77(13), 37-45. https:// doi.org/10.1109/NSSMIC.2009.5401638

Cvejic, N., \& Seppänen, T. (2005). Increasing robustness of LSB audio steganography by reduced distortion LSB coding. Journal of Universal Computer Science, 11(1), 56-65.

Cvejic, N., \& Seppanen, T. (2004). Increasing robustness of LSB audio steganography using a novel embedding method. In 2004 Proceedings of International Conference on Information Technology: Coding and Computing, ITCC 2004 (Vol. 2, pp. 533-537). IEEE Publishing. https://doi.org/10.1109/ ITCC.2004.1286709

Djebbar, F., Ayad, B., Hamam, H., \& Abed-Meraim, K. (2011). A view on latest audio steganography techniques. In 2011 International Conference on Innovations in Information Technology (pp. 409-414). IEEE Publishing. https://doi.org/10.1109/INNOVATIONS.2011.5893859

Gopalan, K. (2018). Audio steganography for information hiding and covert communication - A tutorial. In 2018 IEEE International Conference on Electro/Information Technology (EIT) (pp. 0242-0243). IEEE Publishing. https://doi.org/10.1109/EIT.2018.8500167

Gopalan, K., \& Fu, J. (2015). An imperceptible and robust audio steganography employing bit modification. In 2015 IEEE International Conference on Industrial Technology (ICIT) (pp. 1635-1638). IEEE Publishing. https://doi.org/10.1109/ICIT.2015.7125331

Hameed, A. S. (2018). High capacity audio steganography based on contourlet transform. Tikrit Journal of Engineering Sciences, 25(1), 1-7. https://doi.org/10.25130/tjes.25.01

Hameed, M. A., Hassaballah, M., Aly, S., \& Awad, A. I. (2019). An adaptive image steganography method based on histogram of oriented gradient and PVD-LSB techniques. IEEE Access, 7, 185189-185204. https://doi.org/10.1109/ACCESS.2019.2960254 
Jayapandiyan, J. R., Kavitha, C., \& Sakthivel, K. (2020). Enhanced least significant bit replacement algorithm in spatial domain of steganography using character sequence optimization. IEEE Access, 8, 136537-136545. https://doi.org/10.1109/ACCESS.2020.3009234

Kanhe, A., Aghila, G., Kiran, C. Y. S., Ramesh, C. H., Jadav, G., \& Raj, M. G. (2015). Robust audio steganography based on advanced encryption standards in temporal domain. In 2015 International Conference on Advances in Computing, Communications and Informatics (ICACCI) (pp. 1449-1453). IEEE Publishing. https://doi.org/10.1109/ICACCI.2015.7275816

Kumar, R. (2016). Audio steganography using QR decomposition and fast Fourier transform. Indian Journal of Science and Technology, 6(34), 1-8. https://doi.org/10.17485/ijst/2015/v8i1/69604

Li, P., Kong, Q., \& Ma, Y. (2014). Image secret sharing and hiding with authentication based on PSNR estimation. Journal of Information Hiding and Multimedia Signal Processing, 5(3), 353-366.

Mazdak, Z., Azizah, B. A. M., Shahidan, M. A., \& Saman, S. C. (2012). Mazdak technique for PSNR estimation in audio steganography. In Applied Mechanics and Materials (Vol. 229, pp. 2798-2803). Trans Tech Publications Ltd. https://doi.org/10.4028/www.scientific.net/AMM.229-231.2798

Meligy, A. M., Nasef, M. M., \& Eid, F. T. (2016). A hybrid technique for enhancing the efficiency of audio steganography. International Journal of Image, Graphics and Signal Processing, 8(1), 36-42. https://doi. org/10.5815/ijigsp.2016.01.04

Priyanka, B. R., Vrushabh, K. R., Komal, P. K., Pingle, S. M., \& Mahesh, S. R. (2012). Audio steganography using LSB. International Journal of Electronics, Communication and Soft Computing Science \& Engineering (IJECSCSE), 2, 90-93.

Sapra, P. S. (2016). Secured LSB modification using dual randomness. In 2016 International Conference on Recent Advances and Innovations in Engineering (ICRAIE) (pp. 1-4). IEEE Publishing. https://doi. org/10.1109/ICRAIE.2016.7939592

Somani, H., \& Madhu, K. M. (2015). A survey on digital audio steganography techniques used for secure transmission of data. International Journal of Engineering Development and Research, 3(4), 236-239.

Srivastava, M., \& Rafiq, M. Q. (2012). A novel approach to secure communication using audio steganography. Advanced Materials Research, 408, 963-969. https://doi.org/10.4028/www.scientific.net/AMR.403408.963

Sun, W., Shen, R., Yu, F., \& Lu, Z. (2012). Data hiding in audio based on audio-to-image wavelet transform and vector quantization. In 2012 Eighth International Conference on Intelligent Information Hiding and Multimedia Signal Processing (pp. 313-316). IEEE Publishing. https://doi.org/10.1109/IIH-MSP.2012.82

Tayel, M., Gamal, A., \& Shawky, H. (2016). A proposed implementation method of an audio steganography technique. In 2016 18th international conference on advanced communication technology (ICACT) (pp. 180-184). IEEE Publishing. https://doi.org/10.1109/ICACT.2016.7423320

Wakiyama, M., Hidaka, Y., \& Nozaki, K. (2010). An audio steganography by a low-bit coding method with wave files. In 2010 Sixth International Conference on Intelligent Information Hiding and Multimedia Signal Processing (pp. 530-533). IEEE Publishing. https://doi.org/10.1109/IIHMSP.2010.135 
Xue, P., Liu, H., Hu, J., \& Hu, R. (2018). A multi-layer steganographic method based on audio time domain segmented and network steganography. In AIP Conference Proceedings (Vol. 1967, No. 1, p. 020046). AIP Publishing LLC. https://doi.org/10.1063/1.5039018

Yu, H., Wang, R., Dong, L., Yan, D., Gong, Y., \& Lin, Y. (2020). A high-capacity reversible data hiding scheme using dual-channel audio. IEEE Access, 8, 162271-162278. https://doi.org/10.1109/access.2020.3015851

Zumchak, S. M. (2016). Audio steganography: A comparative study of techniques and tools (Doctoral dissertation). Utica College, USA. 
